\title{
The BET bromodomain inhibitor, JQ1, facilitates C-FLIP degradation and enhances TRAIL-induced apoptosis independent of BRD4 and c-Myc inhibition
}

\author{
Weilong Yao ${ }^{1,2}$, Ping Yue ${ }^{2}$, Fadlo R. Khuri² and Shi-Yong Sun ${ }^{2}$ \\ ${ }^{1}$ Department of Respiration, Xiangya Hospital and Xiangya School of Medicine, Central South University, Changsha, Hunan, \\ PR China \\ ${ }^{2}$ Department of Hematology and Medical Oncology, Winship Cancer Institute, Emory University School of Medicine, Atlanta, \\ Georgia, USA \\ Correspondence to: Shi-Yong Sun, email: ssun@emory.edu
}

Keywords: JQ1, C-FLIP, TRAIL, apoptosis, BRD4, C-Myc

Received: June 20, $2015 \quad$ Accepted: August 31, $2015 \quad$ Published: September 22, 2015

This is an open-access article distributed under the terms of the Creative Commons Attribution License, which permits unrestricted use, distribution, and reproduction in any medium, provided the original author and source are credited.

\section{ABSTRACT}

Inhibition of BET bromodomains (BRDs) has emerged as a promising cancer therapeutic strategy. Accordingly, inhibitors of BRDs such as JQ1 have been actively developed and some have reached clinical testing. However, the mechanisms by which this group of inhibitors exerts their anticancer activity, including induction of apoptosis, have not been fully elucidated. This report reveals a previously uncovered activity of JQ1 in inducing C-FLIP degradation and enhancing TRAILinduced apoptosis. JQ1 potently decreased c-FLIP (both long and short forms) levels in multiple cancer cell lines without apparently increasing the expression of DR5 and DR4. Consequently, JQ1, when combined with TRAIL, synergistically induced apoptosis; this enhanced apoptosis-inducing activity could be abolished by enforced expression of ectopic FLIP or FLIP $_{S}$. Hence it appears that JQ1 decreases C-FLIP levels, resulting in enhancement of TRAIL-induced apoptosis. Inhibition of proteasome with MG132 prevented JQ1-induced C-FLIP reduction. Moreover, JQ1 decreased c-FLIP stability. Therefore, JQ1 apparently decreases c-FLIP levels through facilitating its proteasomal degradation. Genetic inhibition of either BRD4 or c-Myc by knocking down their expression failed to mimic JQ1 in decreasing C-FLIP and enhancing TRAILinduced apoptosis, suggesting that JQ1 induces C-FLIP degradation and enhances TRAIL-induced apoptosis independent of BRD4 or c-Myc inhibition. In summary, our findings in this study highlights a novel biological function of JQ1 in modulating apoptosis and warrant further study of the potential treatment of cancer with the JQ1 and TRAIL combination.

\section{INTRODUCTION}

The bromodomain (BRD) and extra-terminal domain (BET) family is comprised of four proteins: $\mathrm{BRD} 2, \mathrm{BRD} 3, \mathrm{BRD} 4$, and BRDT, which perform diverse roles in regulating gene transcription. These BET family proteins have been identified in oncogenic rearrangements, generating highly oncogenic fusion proteins, and in regulating transcription of several oncogenes, such as c-Myc and Bcl-2. Therefore they play key roles in oncogenesis of certain types of cancer and accordingly, targeting BET proteins has emerged as a promising cancer therapeutic strategy $[1,2]$.

In the past few years, several small molecule inhibitors that target BET family proteins, particularly BRD4, have been developed. These inhibitors have been actively used either as therapeutic agents or as research tools in many preclinical studies and some of them have advanced to testing in clinical trials $[1,2]$. JQ1, a triazolothienodiazepine (Figure 1A), is the first BET BRD inhibitor developed for cancer therapy $[3,4]$ and hence has been widely used. One initial putative mechanism by which JQ1 and other BET BRD inhibitors exert their anticancer activity is through suppression of c-Myc 
expression [3, 5]. However, other mechanisms involving regulation of a different set of cancer-relevant genes independent of c-Myc have also been suggested [6, 7]. By far, the majority of studies have shown that the therapeutic effect of BET BRD inhibitors is attributed to targeting $\mathrm{BRD} 4$, rather than the other BET proteins [2].

JQ1 and other BET BRD inhibitors have been reported to induce apoptosis in some types of cancer cells including lung cancer cells, contributing to their anticancer activity [8-14]. However, the underlying mechanisms of apoptosis induction are largely unclear other than Bim involvement $[10,12]$. In addition to single agent activity, these inhibitors also act synergistically with other cancer therapeutic agents such as HDAC inhibitors [15, 16], mTOR inhibitors [13, 17, 18], and PI3K inhibitors [19] in inducing apoptosis and inhibiting the growth of cancer cells and tumors. Further evaluation of these combinations in the clinic is thus warranted even though continuous investigation of their biological actions is still needed.

Tumor necrosis factor-related apoptosis-inducing ligand (TRAIL; also called APO-2L) is a well-known death ligand or cytokine that initiates apoptosis upon ligation with two death receptors named death receptor 4 (DR4) and 5 (DR5). TRAIL preferentially induces apoptosis in transformed or malignant cells, but not in most normal cells, and thus is a tumor-selective apoptosisinducing cytokine with cancer therapeutic potential [20, 21]. Recombinant human TRAIL is currently being tested in clinical trials as a potential cancer therapeutic agent [2123]. Unfortunately cancer cells exhibit varied sensitivity to TRAIL and a substantial proportion of cancer cell lines are intrinsically insensitive to TRAIL [20]. Thus, additional sensitization is needed to potentiate the killing effect of TRAIL in these insensitive cancer cells.

Cellular FLICE-inhibitory protein (c-FLIP), a truncated form of caspase- 8 that lacks enzymatic activity, is known to be a negative regulator of TRAIL-induced apoptosis by blocking the activation of caspase- 8 through competing with caspase-8 for binding to FADD [24]. Numerous studies have documented that elevated c-FLIP expression protects cells from TRAIL-mediated apoptosis, whereas downregulation of c-FLIP by chemicals or small interfering RNA (siRNA) sensitizes cells to TRAILinduced apoptosis [25]. Elevated levels of c-FLIP have been found in a number of different cancers and are often correlated with a poor prognosis in certain types of cancers [26]. Hence, downregulation of c-FLIP is an effective strategy to sensitize cancer cells to TRAIL-induced apoptosis.

The current study has revealed a previously uncovered biological function of JQ1 and other BET BRD inhibitors in potently decreasing c-FLIP levels and sensitizing cancer cells to TRAIL-induced apoptosis. However these effects are not mediated by inhibition of either BRD4 or c-Myc.

\section{RESULTS}

\section{JQ1 decreases c-FLIP levels in cancer cell lines}

Human non-small cell lung cancer (NSCLC) cell lines display varied sensitivities to JQ1 treatment $[6,14]$, with some being quite resistant [6]. In an effort to identify agents that suppress c-FLIP expression, we found that JQ1 at a range of 1 to $5 \mu \mathrm{M}$ effectively decreased the levels of c-FLIP including both long $\left(\right.$ FLIP $\left._{\mathrm{L}}\right)$ and short $\left(\right.$ FLIP $\left._{\mathrm{S}}\right)$ forms in the 3 tested JQ1-senstive NSCLC cell lines, H157, H1299 and A549 (Figures. 1B and 1C). Apparent reduction of c-FLIP occurred at $3 \mathrm{~h}$ post JQ1 treatment and was sustained up to $16 \mathrm{~h}$ (Figure 1D). Under the tested conditions, JQ1 did not obviously increase DR5 expression and decrease the levels of Mcl-1, Bcl- $\mathrm{X}_{\mathrm{L}}$ and survivin in any of the tested cell lines. JQ1 weakly decreased Bcl-2 levels in H1299 and A549 cells, but not in H157 cells. Interestingly, JQ1 decreased DR4 expression, particularly in H157 and H1299 cells. JQ1 partially decreased c-Myc levels in H1299 cells, but increased its levels in H157 and A549 cells (Figure 1C). Hence, JQ1 effectively decreases c-FLIP levels in cancer cells.

\section{JQ1 synergizes with TRAIL to induce apoptosis}

Next we determined whether JQ1 sensitizes cancer cells to TRAIL-induced apoptosis due to its c-FLIPreducing activity. In two TRAIL-insensitive cell lines, A549 and H1299, the combination of JQ1 and TRAIL was much more active than either agent alone in decreasing the survival of cancer cells (Figure 2A). The combination indexes (CIs) for every combination were far less than 1 , indicating strong synergy. In agreement, the combination of JQ1 and TRAIL was much more potent than either single agent alone in inducing cleavage of caspase-8, caspase- 3 and PARP, hallmarks of apoptosis, and in enhancing Annexin V-positive apoptotic cells in both A549 and H1299 cells (Figures. 2B and 2C). Together these results robustly indicate that the JQ1 and TRAIL combination displays synergistic effect in the induction of apoptosis.

\section{Enforced ectopic c-FLIP overexpression abolishes synergistic induction of apoptosis by the JQ1 and TRAIL combination}

To explore the role of c-FLIP downregulation in the synergistic induction of apoptosis by the JQ1 and TRAIL combination, we compared the effects of JQ1 plus TRAIL on apoptosis induction in H157 cell lines that express ectopic Lac Z (as a control), FLIP ${ }_{\mathrm{L}}$ or FLIP $_{\mathrm{S}}$ (Figure 3A). Both JQ1 and TRAIL alone minimally or 
weakly decreased cell survival and induced cleavage of caspase-8, caspase-3 and PARP in H157-Lac Z cells. The combination of JQ1 and TRAIL, however, exerted synergistic effects on decreasing cell survival (CIs $<1$ ) and inducing caspase cleavage in this cell line. These synergistic effects were lost in H157-FLIP ${ }_{L}$ and H157-
FLIP $_{\mathrm{S}}$ cells (Figures. 3B and 3C). Similar results were also observed in A549 cells that express ectopic FLIP $_{\mathrm{L}}$ (data not shown). These data thus clearly suggest that c-FLIP downregulation is critical for the enhancement of TRAILinduced apoptosis by JQ1.
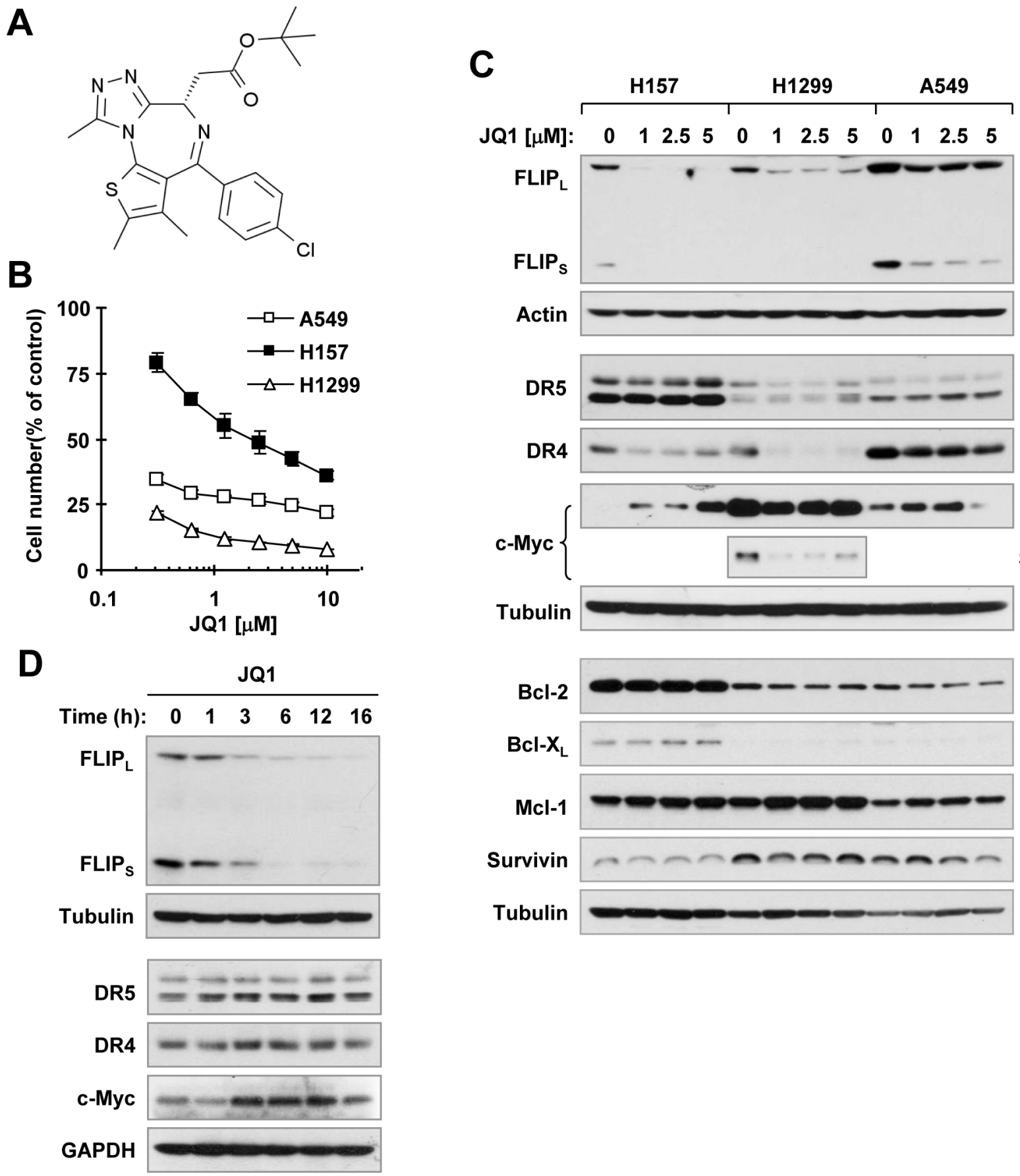

Figure 1: JQ1 (A) decreases the survival of cancer cells (B) and decreases c-FLIP levels (C and D). A, Chemical structure of JQ1. B, The indicated cancer cell lines were treated with different concentrations of JQ1 for 3 days and then subjected to estimation of cell number with the SRB assay. The data are means \pm SDs of four replicate determinations. $\mathbf{C}$ and $\mathbf{D}$, The indicated cell lines were treated with the given concentrations of JQ1 for $12 \mathrm{~h}(\mathbf{C})$ or $3 \mu \mathrm{M}$ for the different times as indicated (D) and then harvested for preparation of whole-cell protein lysates and subsequent Western blot analysis. SE, short exposure. 


\section{JQ1 promotes c-FLIP protein degradation}

Given that c-FLIPs are unstable proteins subjected to proteasomal degradation [27, 28], we then determined whether JQ1 facilitates proteasomal degradation of c-FLIP, causing c-FLIP reduction in cancer cells. We found that the presence of the proteasome inhibitor MG132 prevented both forms of c-FLIP from reduction induced by JQ1 (Figure 4A). Moreover, the half-lives of both FLIP $_{\mathrm{L}}$ and FLIP $\mathrm{S}_{\mathrm{S}}$ in JQ1-treated cells were shorter than those in DMSO-treated cells (Figure 4B), indicating that JQ1 facilitates c-FLIP degradation or decreases their stability. Collectively these results suggest that JQ1 decreases the levels of c-FLIPs involving facilitation of their degradation.

JQ1-induced c-FLIP reduction and enhancement of TRAIL-induced apoptosis are independent of BRD4 and c-Myc inhibition

One putative mechanism of action of BET BRD inhibitors involves suppression of c-Myc [3,5]. To determine whether JQ1-induced c-FLIP reduction is the consequence of BRD4 or c-Myc inhibition, we first analyzed the effects of other BET BRD inhibitors on
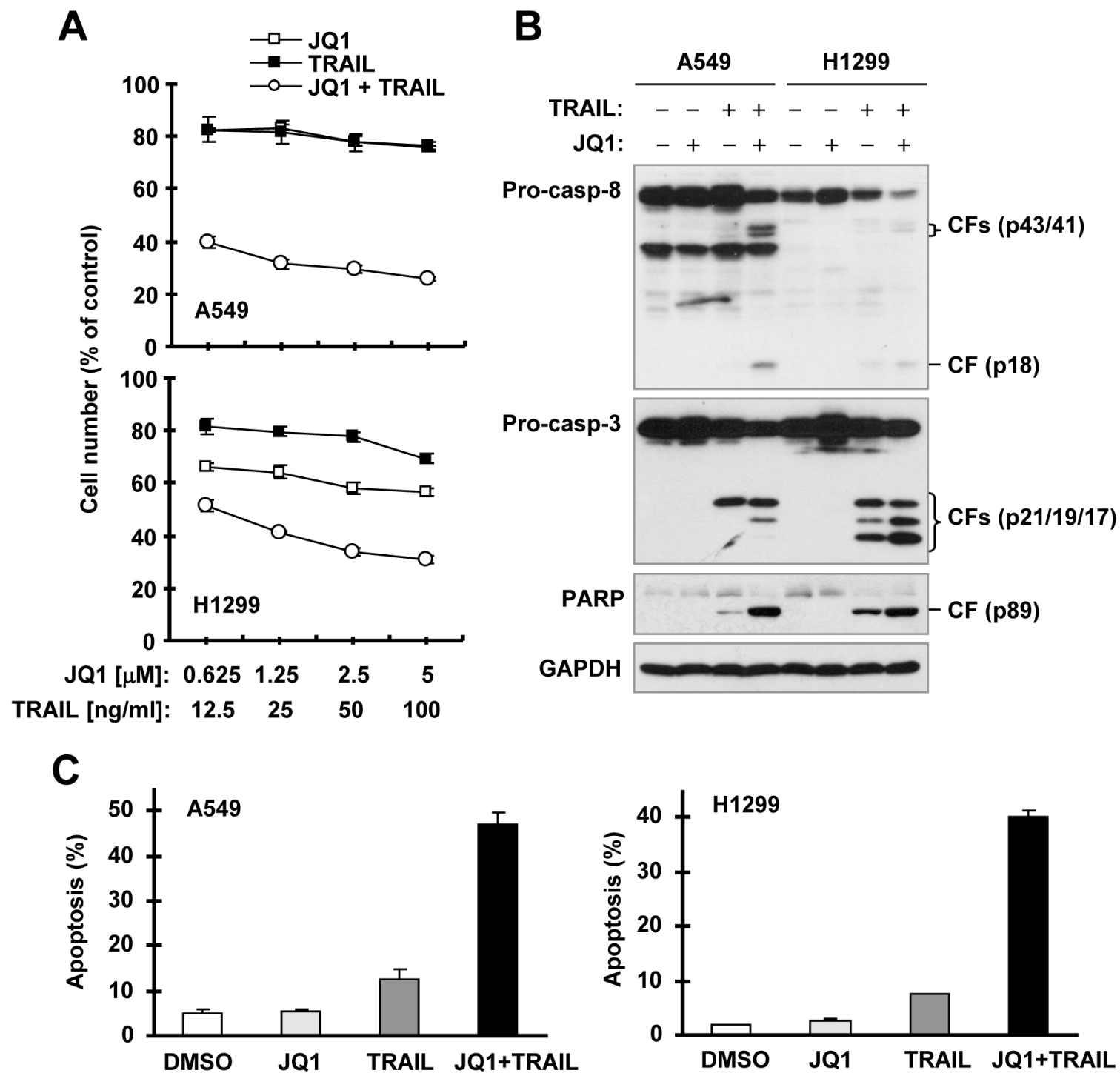

Figure 2: JQ1 synergizes with TRAIL to augment killing of cancer cells and induction of apoptosis (B and C). A, The indicated cancer cell lines were treated with different concentrations of JQ1 alone, TRAIL alone, and the combination of JQ1 and TRAIL for $24 \mathrm{~h}$ and then subjected to estimation of cell number with the SRB assay. The data are means \pm SDs of four replicate determinations. $\mathbf{B}$ and $\mathbf{C}$, The indicated cell lines were treated with $1.25 \mu \mathrm{M}$ JQ1 alone, $25 \mathrm{ng} / \mathrm{ml}$ TRAIL alone or their combination for $14 \mathrm{~h}$ and then subjected to preparation of whole-cell protein lysates and subsequent Western blot analysis (B) and to detection of apoptosis with annexin V-PE and 7AAD staining followed by flow cytometric analysis (C). CF, cleaved fragment. 
altering c-FLIP levels and enhancing TRAIL-induced apoptosis. OTX015, a compound highly related to JQ1 in chemical structure (Figure 5A), was as effective as JQ1 in decreasing the levels of c-FLIP (Figure 5B). PFI-1 and MS436, which have distinct chemical structures from JQ1, either weakly decreased (PFI-1) or even slightly increased (MS436) c-FLIP levels (Figure 5B). Consistently, OTX05, but not PFI-1 and MS436, enhanced TRAILinduced apoptosis, as evidenced by increased cleavage of caspase-8, caspase-3 and PARP, as did JQ1 (Figure 5C).

Moreover, we used a more specific genetic approach by directly knocking down BRD4 or c-Myc and investigating the impact of their knockdown on c-FLIP levels and TRAIL-induced apoptosis. Knockdown of BRD4 actually slightly increased c-FLIP levels in A549 and H157 cells, but slightly decreased c-FLIP levels in H1299 and HCC827 cells (Figure. 6A). Similarly, knockdown of c-Myc increased c-FLIP levels in H1299 and A549 cells, but decreased c-FLIP levels in H157 cells (Figure 6B). Hence it is clear that knockdown of both BRD4 and c-Myc generates mixed effects on c-FLIP levels depending on cell lines. Moreover, knockdown of either BRD4 or c-Myc did not apparently enhance the potency of TRAIL in decreasing cell survival (Figure 6C) and in inducing cleavage of caspase- 8 , caspase- 3 and PARP (Figure 6D) in both H1299 and A549 cells, indicating that genetic suppression of BRD4 or c-Myc did not accordingly sensitize cancer cells to TRAIL-induced apoptosis. Taking these data together, we suggest that genetic inhibition of BRD4 or c-Myc does not mimic

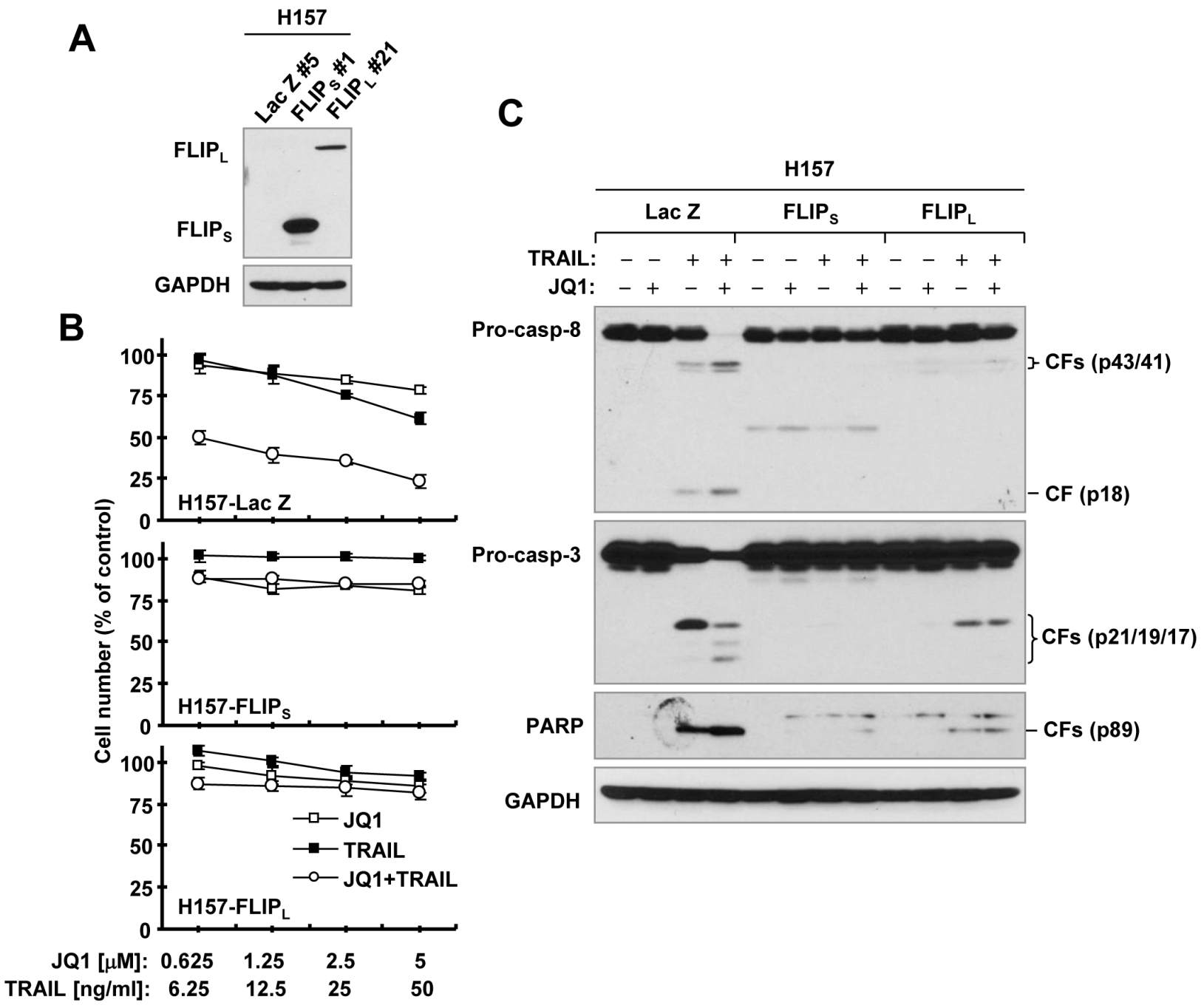

Figure 3: Enforced expression of ectopic c-FLIP (A) abolishes the synergistic effects of JQ1 and TRAIL on decreasing cell survival (B) and activating caspase cascade (C). A, Ectopic expression of c-FLIP was verified with Western blotting. B, The indicated cell lines were treated with different concentrations of JQ1 alone, TRAIL alone, and the combination of JQ1 and TRAIL for $24 \mathrm{~h}$ and then subjected to estimation of cell number with the SRB assay. The data are means \pm SDs of four replicate determinations. C, The indicated cell lines were treated with $1 \mu \mathrm{M}$ JQ1 alone, $20 \mathrm{ng} / \mathrm{ml}$ TRAIL alone or their combination for $12 \mathrm{~h}$ and then subjected to preparation of whole-cell protein lysates and subsequent Western blot analysis. CF, cleaved fragment. 
the ability of JQ1 to decrease c-FLIP levels and enhance TRAIL-induced apoptosis.

\section{Inhibition of c-Myc elevation fails to abolish the ability of JQ1 to reduce c-FLIP levels}

In our study, we found that JQ1 increased the levels of c-Myc in A549 and H157 cells as presented in Figure 1. A previous study has suggested that c-Myc directly suppresses c-FLIP gene expression [29]. To determine whether JQ1-induced c-FLIP reduction is connected to c-Myc upregulation in these two cell lines, we used c-Myc siRNA transfection to knock down c-Myc expression including c-Myc upregulation induced by JQ1 and then examined its impact on c-FLIP reduction induced by JQ1. We found that c-Myc siRNA effectively decreased c-Myc expression, but failed to prevent c-FLIP reduction induced by JQ1 although the basal levels of c-FLIP were elevated by c-Myc knockdown in A549 cells (Figure 7), suggesting that JQ1-induced c-FLIP reduction is unlikely secondary to c-Myc upregulation in these cell lines.

\section{DISCUSSION}

The current study has shown that JQ1 decreases c-FLIP levels and enhances TRAIL-induced apoptosis in cancer cells, uncovering a novel biological function of JQ1 in the regulation of the extrinsic apoptotic pathway. In this study, JQ1 substantially decreased c-FLIP (both FLIP and FLIP $_{\mathrm{S}}$ ) levels without increasing the expression of DR4 and DR5, two other critical components in mediating TRAIL-induced apoptosis [22]. Moreover, enforced expression of ectopic c-FLIP abrogates the ability of JQ1 to enhance TRAIL-induced apoptosis. Therefore, it is very likely that JQ1 synergizes with TRAIL to augment apoptosis primarily through c-FLIP downregulation. Although suppression of other anti-apoptotic proteins such as Bcl-2 family members and survivin sensitizes cancer cells to TRAIL-induced apoptosis [30], JQ1 did not decrease the levels of Bcl- $\mathrm{X}_{\mathrm{L}}, \mathrm{Mcl}-1$ and survivin in every tested cell lines except for weak effects on reducing Bcl-2 in H1299 and A549 cells. Therefore, JQ1induced enhancement of TRAIL-induced apoptosis is not associated with modulation of these proteins.

Protein ubiquitinylation and proteasomal
A

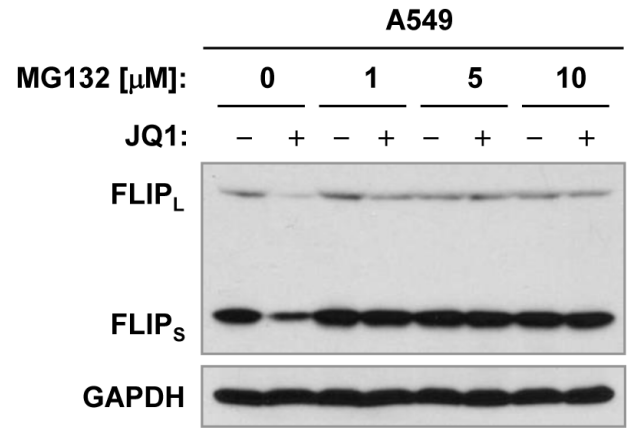

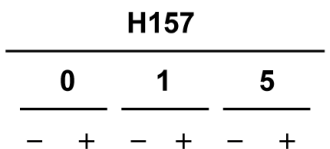

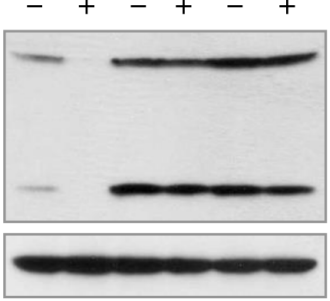

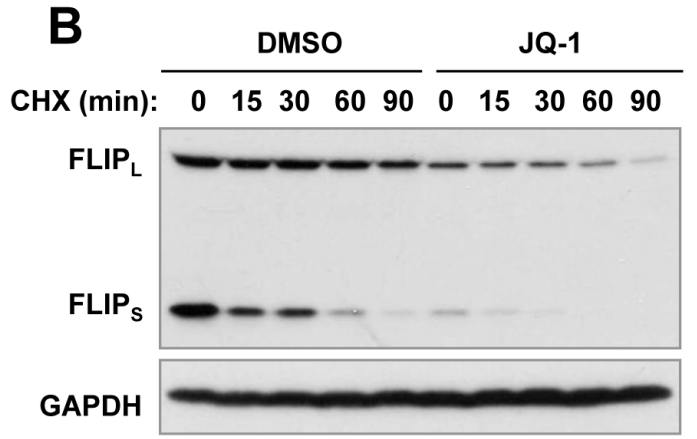

GAPDH
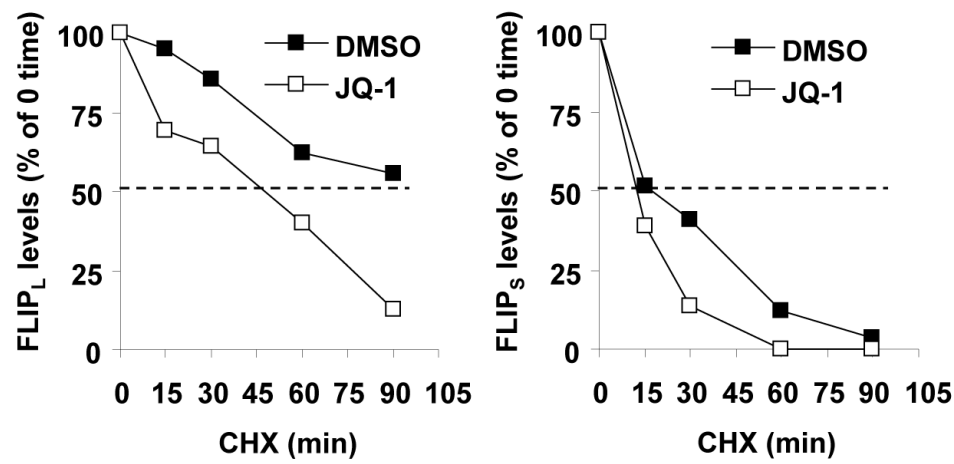

Figure 4: JQ1 decreases levels of c-FLIPs through facilitating their degradation. A, The indicated cell lines were pretreated with the indicated different concentrations of MG132 for 30 minutes prior to the addition of $1 \mu \mathrm{M} \mathrm{JQ1}$. After co-treatment for 4 h, the cells were harvested for preparation of whole-cell protein lysates and subsequent Western blot analysis. B, A549 cells were treated with DMSO or $1 \mu \mathrm{M} \mathrm{JQ} 1$ for $10 \mathrm{~h}$. The cells were then washed with PBS 3 times and refed with fresh medium containing $10 \mu \mathrm{g} / \mathrm{ml} \mathrm{CHX.} \mathrm{At} \mathrm{the}$ indicated times, the cells were harvested for preparation of whole-cell protein lysates and subsequent Western blot analysis. Protein levels were quantitated with NIH Image J software (Bethesda, MA) and were normalized to actin. The results were plotted as the relative c-FLIP levels compared to those at the time 0 of CHX treatment (right panels). 
degradation is a key mechanism that regulates c-FLIP levels $[25,31]$. In this study, inhibition of the proteasome with MG132 rescued c-FLIP downregulation caused by JQ1. Moreover JQ1 treatment destabilized c-FLIP proteins by facilitating their degradation. Hence it is clear that JQ1 decreases c-FLIP levels by promoting their proteasomal degradation.

The majority of studies to date have shown that the therapeutic effect of BET BRD inhibitors is largely due to targeting BRD4, rather than the other BET proteins [2]. Since JQ1 is primarily a BRD4 inhibitor [4], it is reasonable to ask whether inhibition of BRD4 accounts for c-FLIP reduction induced by JQ1. Further study with additional BET BRD inhibitors including OTX015, PFI1 and MS436 found that OTX015, but not PFI-1 and MS436, displayed similar effects as JQ1 did in decreasing c-FLIP levels and enhancing TRAIL-induced apoptosis. While PFI-1 and MS436 are selective BRD4 inhibitors [9, 32], OTX015 is an inhibitor of BRD2, BRD3 and BRD4
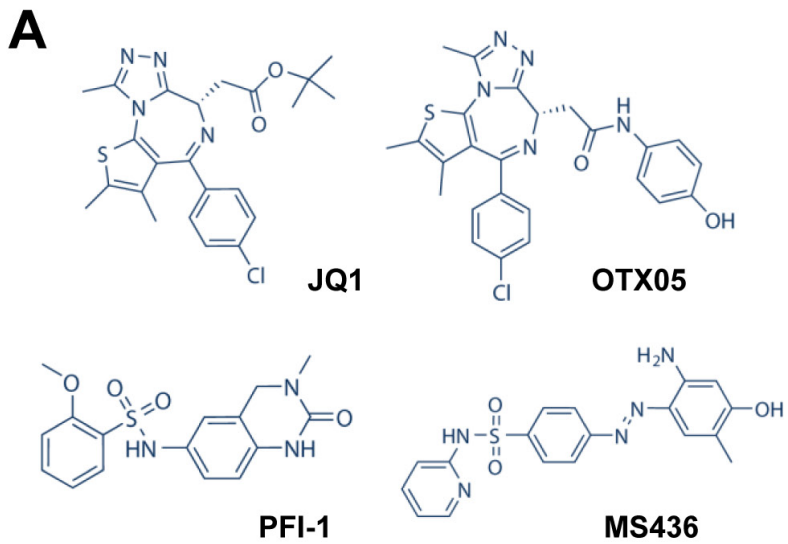

B

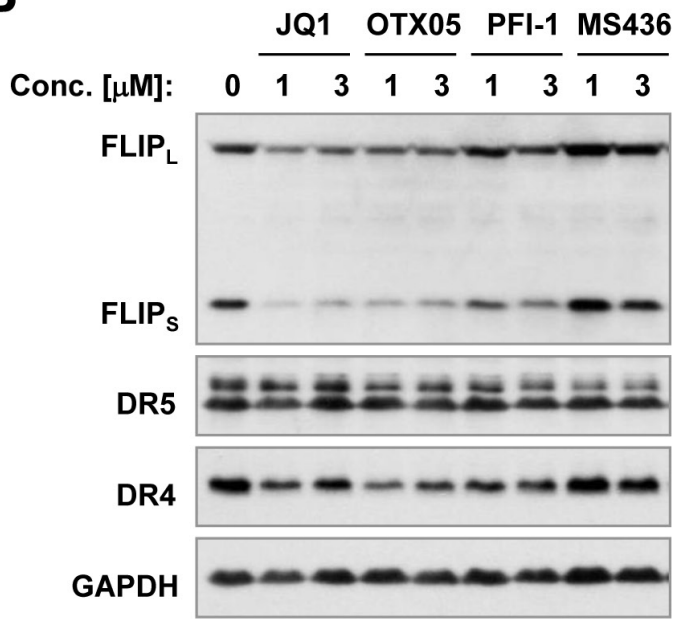

[33]. However, OTX015 is chemically closer to JQ1 because they share similar parental chemical structures, while PFI-1 and MS436 have relatively distinct chemical structures (Figure 6A). Interestingly, it is OTX015, but not PFI-1 and MS432, that functions as effectively as JQ1 in decreasing c-FLIP levels and enhancing TRAIL-induced apoptosis. It seems that JQ1 and OTX015 share similar activity in modulating c-FLIP levels due to their chemical similarity, rather than inhibition of BRD4. Suppression of BRD4 with siRNA-mediated gene knockdown did not result in substantial reduction of c-FLIP and enhancement of TRAIL-induced apoptosis. Instead, we observed an increase in c-FLIP in some cell lines. Hence, the genetic suppression of BRD4 fails to mimic the effect of JQ1 in decreasing c-FLIP and sensitizing cancer cells to TRAILinduced apoptosis. Collectively, we conclude that JQ1 decreases c-FLIP levels and enhances TRAIL-induced apoptosis independent of BRD4 inhibition.

While c-Myc has been suggested to be a putative

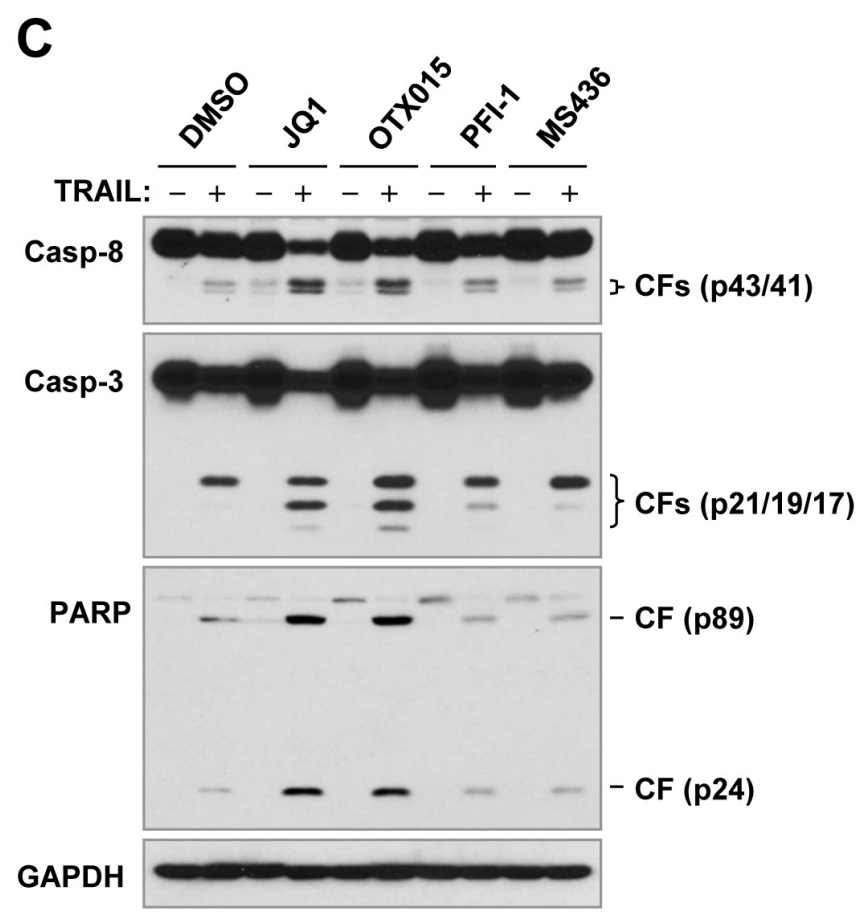

Figure 5: Effects of different BRD inhibitors (A) on decreasing c-FLIP levels (B) and on enhancing TRAIL-induced apoptosis (C). A, Chemical structures of the tested BRD inhibitors. B and C, A549 cells were exposed to the different concentrations of inhibitors as indicated for $8 \mathrm{~h}(\mathbf{B})$ or treated with $1 \mu \mathrm{M}$ of a given BRD inhibitor alone, $50 \mathrm{ng} / \mathrm{ml}$ TRAIL alone or their respective combinations for $14 \mathrm{~h}(\mathbf{C})$. The cells were then harvested for preparation of whole-cell protein lysates and subsequent Western blot analysis for detection of the indicated proteins. CF, cleaved fragment. 
A

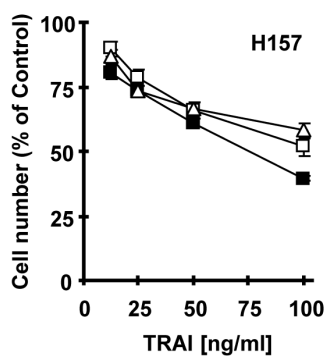

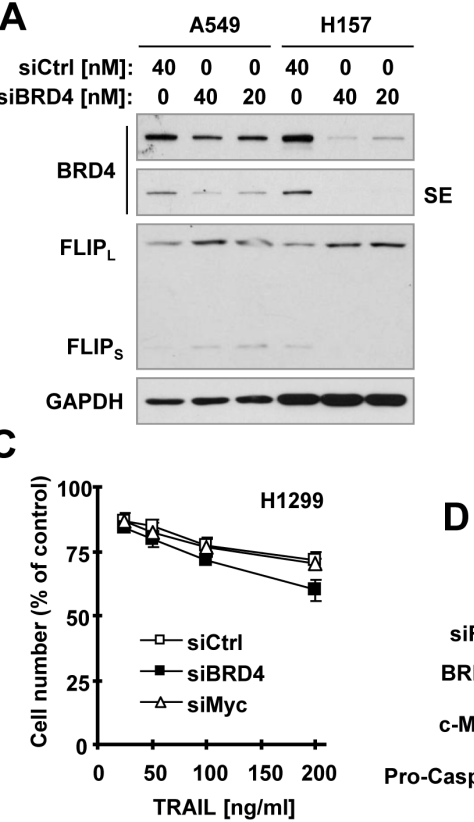

B

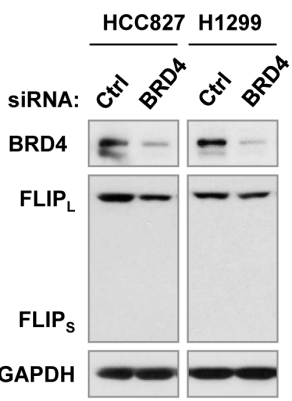

B

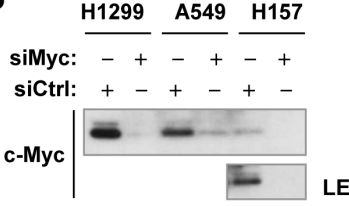

FLIP $_{\mathrm{L}}$
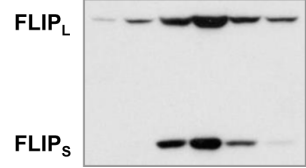

GAPDH

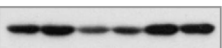

D $\quad$ H1299

H157
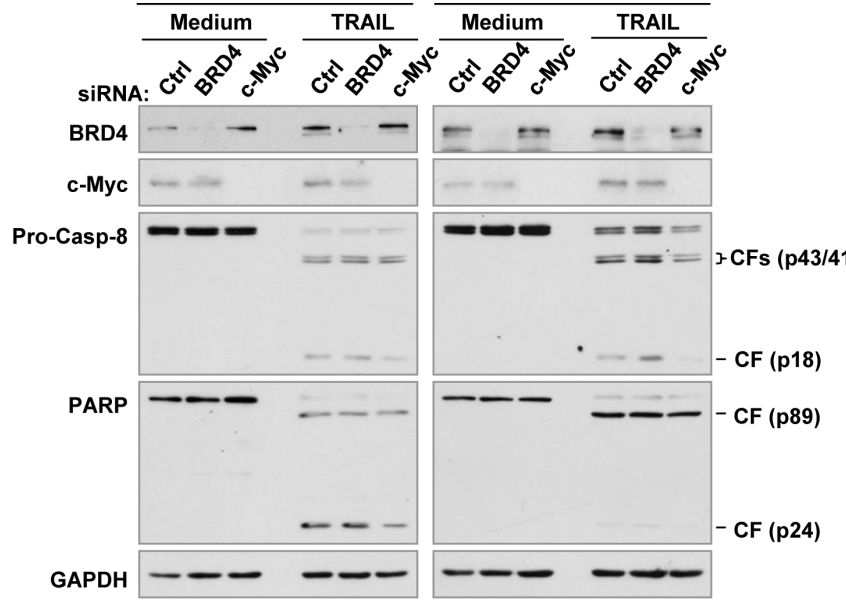

Figure 6: Effects of siRNA-mediated genetic suppression of BRD4 or c-Myc on modulating c-FLIP levels (A and B) and TRAIL-induced apoptosis (C and D). A and B, The indicated cancer cell lines were transfected with control (Ctrl), BRD4 or c-Myc siRNA for $48 \mathrm{~h}$ and then harvested for preparation of whole-cell protein lysates and subsequent Western blot analysis. C and $\mathbf{D}$, The indicated cell lines were transfected with control (Ctrl), BRD4 or c-Myc siRNA for $24 \mathrm{~h}$ and re-plated in 96-well plates and 6-well plates. On the second day, the cells in 96-well plates (C) were treated with different concentrations of TRAIL for $24 \mathrm{~h}$ and then subjected to estimation of cell number with the SRB assay. The data are means \pm SDs of four replicate determinations. The cells in 6-well plates (D) were treated with $100 \mathrm{ng} / \mathrm{ml}$ (H1299) or $50 \mathrm{ng} / \mathrm{ml}$ (H157) TRAIL for $24 \mathrm{~h}$ and then subjected to preparation of whole-cell protein lysates and subsequent Western blot analysis. CF, cleaved fragment.

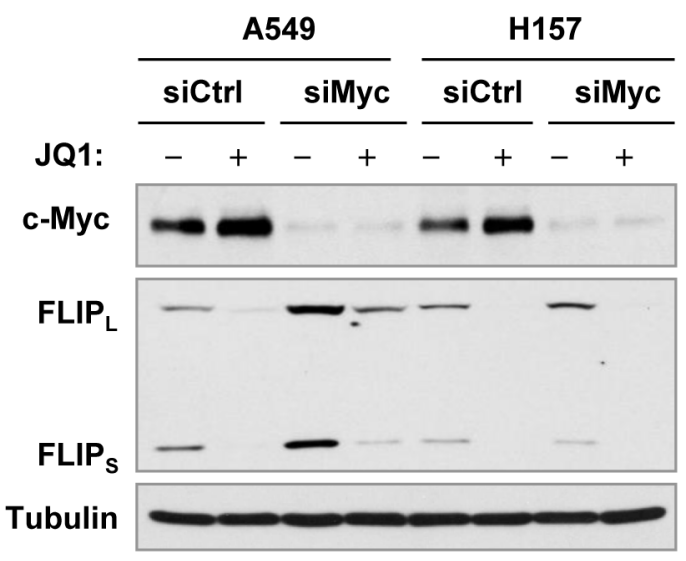

Figure 7: Effects of c-Myc knockdown on JQ1-induced c-FLIP reduction in cancer cell lines in which c-Myc expression is increased by JQ1 treatment. The indicated cell lines were transfected with control (Ctrl) or c-Myc siRNA (siMyc) for $38 \mathrm{~h}$ and then exposed to DMSO or $2 \mu \mathrm{M}$ JQ1 for additional $12 \mathrm{~h}$. The cells were then harvested for preparation of whole-cell protein lysates and subsequent Western blot analysis. 
target gene that mediates the cancer therapeutic activity of JQ1 and other BET BRD inhibitors [2, 3, 5], increased recent studies have suggested that these inhibitors exert c-Myc-independent activity [2, 6, 7]. In our study, JQ1 in fact increased c-Myc levels in H157 and A549 cells but decreased its levels in H1299 cells. This finding is in agreement with a previous study that shows c-Myc upregulation by JQ1 in some lung cancer cell lines [6]. Genetic suppression of c-Myc through siRNA-mediated gene knockdown did not substantially decrease c-FLIP levels, rather increased c-FLIP levels in some cancer cell lines (e.g., A549 and H1299). Moreover we did not find that genetic suppression of c-Myc mimicked the ability of JQ1 to enhance TRAIL-induced apoptosis. Therefore it is unlikely that JQ1 decreases c-FLIP levels and enhances TRAIL-induced apoptosis through c-Myc suppression.

A previous study has suggested that c-Myc suppresses c-FLIP by directly repressing its transcription [29]. In agreement, we found that c-Myc knockdown indeed elevated basal levels of c-FLIP in some cell lines (e.g., A549 and H1299). The c-Myc elevation in some cell lines (e.g., A549 and H157) exposed to JQ1 raised the possibility that c-FLIP reduction by JQ1 is secondary to c-Myc upregualtion, at least in these cell lines. However, suppression of c-Myc upregulation by knocking down its expression failed to affect the ability of JQ1 to decrease c-FLIP levels in both A549 and H157 cells although it elevated basal levels of c-FLIP in A549 cells. Combining with the data that JQ1 decreased the levels of both c-FLIP and c-Myc in H1299 cells, we suggest that c-FLIP reduction induced by JQ1 is unlikely due to c-Myc upregulation either.

Hence, the mechanism by which JQ1 decrease c-FLIP levels via facilitating their degradation has not been elucidated in this study and thus deserves further investigation in the future. The general mechanisms accounting for degradation of both FLIP $_{L}$ and FLIP $_{S}$ have not been fully elucidated by now. Our findings in this study suggest that JQ1 can be a valuable research tool for understanding c-FLIP degradation mechanisms.

Targeting the TRAIL/death receptor signaling pathway with either recombinant TRAIL or agonistic death receptor antibodies has been considered a promising cancer therapeutic strategy and has been tested in the clinic [20-23]. Our findings warrant further investigation of JQ1 or OTX015 as a sensitizer of TRAIL/DR-targeted cancer therapy in vivo and in the clinic, thus promising the clinical translational significance of this approach.

\section{MATERIALS AND METHODS}

\section{Reagents}

(+)-JQ1 was purchased from ApexBio (Houston, TX). OTX015 and MS436 were purchased from Cayman Chemical (Ann Arbor, MI). Soluble recombinant human TRAIL was purchased from PeproTech, Inc. (Rocky Hill, NJ). MG132, cycloheximide (CHX) and PFI-1 were purchased from Sigma Chemical Co. (St. Louis, MO). Monoclonal anti-FLIP antibody (NF6) was obtained from Alexis Biochemicals (San Diego, CA). Mouse monoclonal caspase-8, PARP, survivin and c-Myc antibodies were purchased from Cell Signaling Technology, Inc. (Danvers, MA). Mouse monoclonal caspase-3 antibody was purchased from Imgenex (San Diego, CA). Rabbit polyclonal DR5 antibody was obtained from ProSci Inc. (Poway, CA). Mouse monoclonal DR4 antibody (B-N28) was purchased from Diaclone (Stamford, CT). Rabbit polyclonal Mcl-1 and Bcl- $\mathrm{X}_{\mathrm{L} / \mathrm{S}}$ and mouse monoclonal Bcl-2 antibody were purchased from Santa Cruz Biotechnology, Inc. (Santa Cruz, CA). Tubulin and GAPDH antibodies were purchased from Sigma Chemical Co. and Trevigen Inc. (Gaithersburg, MD), respectively.

\section{Cell lines and cell culture}

Human cancer cell lines used in this study were described in our previous work [34]. Except for H157 and A549 cells, which were authenticated by Genetica DNA Laboratories, Inc. (Cincinnati, OH) through analyzing short tandem repeat DNA profile, other cell lines have not been authenticated. The stable cell lines, H157-Lac Z \#5, H157-FLIP $\# 1$ and H157-FLIP $\# 21$ were described previously [35, 36]. A549-Lac Z \#2, A549-Lac Z \#9 and $\mathrm{A}_{549-\text { FLIP }_{L}} \# 4$ were described in our previous studies [37, 38]. These cell lines were cultured in RPMI 1640 medium containing $5 \%$ fetal bovine serum at $37^{\circ} \mathrm{C}$ in a humidified atmosphere of $5 \% \mathrm{CO}_{2}$ and $95 \%$ air.

\section{Cell survival and apoptosis assays}

Cells were seeded in 96-well cell culture plates and treated the next day with the given agents. Viable cell numbers were determined using sulforhodamine B (SRB) assay as described previously [39]. CI for drug interaction (e.g., synergy) was calculated using the CompuSyn software (ComboSyn, Inc.; Paramus, NJ). Apoptosis was evaluated by detection of caspase and PARP cleavage with Western blot analysis and with a PE Annexin V Apoptosis Detection kit (BD Biosciences; San Jose, CA) according to the manufacturer's instructions. 


\section{Western blot analysis}

Preparation of whole-cell protein lysates and performance of the Western blot analysis were the same as described previously [40].

\section{Gene knockdown by siRNA}

BRD4 (sc-43639) and c-Myc (\#6341) siRNAs were purchased from Santa Cruz Biotechnology (Santa Cruz, CA) and Cell Signaling Technology, Inc., respectively. Transfection of these siRNA duplexes was conducted in 6-well plates using the HiPerFect transfection reagent (Qiagen) following the manufacturer's manual.

\section{ACKNOWLEDGMENTS}

We are grateful to Dr. Anthea Hammond in our department for editing the manuscript. We are also thankful to the high school student, Jerry Yue, from Parkview High School (Lilburn, GA) for assisting performance of some experiments.

This study was supported by the Emory Winship Cancer Institute Halpern Research Scholar award (to SYS).

FR Khuri and S-Y Sun are Georgia Research Alliance Distinguished Cancer Scientists. S-Y Sun is a Halpern Research Scholar.

\section{CONFLICTS OF INTEREST}

There is no conflict of interest.

\section{REFERENCES}

1. Fu LL, Tian M, Li X, Li JJ, Huang J, Ouyang L, Zhang $\mathrm{Y}$ and Liu B. Inhibition of BET bromodomains as a therapeutic strategy for cancer drug discovery. Oncotarget. 2015; 6:5501-5516.

2. Shi J and Vakoc CR. The mechanisms behind the therapeutic activity of BET bromodomain inhibition. Mol Cell. 2014; 54:728-736.

3. Delmore JE, Issa GC, Lemieux ME, Rahl PB, Shi J, Jacobs HM, Kastritis E, Gilpatrick T, Paranal RM, Qi J, Chesi M, Schinzel AC, McKeown MR, Heffernan TP, Vakoc CR, Bergsagel PL, et al. BET bromodomain inhibition as a therapeutic strategy to target c-Myc. Cell. 2011; 146:904917.

4. Filippakopoulos P, Qi J, Picaud S, Shen Y, Smith WB, Fedorov O, Morse EM, Keates T, Hickman TT, Felletar I, Philpott M, Munro S, McKeown MR, Wang Y, Christie AL, West N, et al. Selective inhibition of BET bromodomains. Nature. 2010; 468:1067-1073.

5. Mertz JA, Conery AR, Bryant BM, Sandy P,
Balasubramanian S, Mele DA, Bergeron L and Sims RJ, 3rd. Targeting MYC dependence in cancer by inhibiting BET bromodomains. Proc Natl Acad Sci U S A. 2011; 108:16669-16674.

6. Lockwood WW, Zejnullahu K, Bradner JE and Varmus H. Sensitivity of human lung adenocarcinoma cell lines to targeted inhibition of BET epigenetic signaling proteins. Proc Natl Acad Sci U S A. 2012; 109:19408-19413.

7. Baker EK, Taylor S, Gupte A, Sharp PP, Walia M, Walsh NC, Zannettino AC, Chalk AM, Burns CJ and Walkley CR. BET inhibitors induce apoptosis through a MYC independent mechanism and synergise with CDK inhibitors to kill osteosarcoma cells. Scientific reports. 2015; 5:10120.

8. Puissant A, Frumm SM, Alexe G, Bassil CF, Qi J, Chanthery YH, Nekritz EA, Zeid R, Gustafson WC, Greninger P, Garnett MJ, McDermott U, Benes CH, Kung AL, Weiss WA, Bradner JE, et al. Targeting MYCN in neuroblastoma by BET bromodomain inhibition. Cancer Discov. 2013; 3:308-323.

9. Picaud S, Da Costa D, Thanasopoulou A, Filippakopoulos P, Fish PV, Philpott M, Fedorov O, Brennan P, Bunnage ME, Owen DR, Bradner JE, Taniere P, O'Sullivan B, Muller S, Schwaller J, Stankovic T, et al. PFI-1, a highly selective protein interaction inhibitor, targeting BET Bromodomains. Cancer Res. 2013; 73:3336-3346.

10. Patel AJ, Liao CP, Chen Z, Liu C, Wang Y and Le LQ. BET bromodomain inhibition triggers apoptosis of NF1associated malignant peripheral nerve sheath tumors through Bim induction. Cell Rep. 2014; 6:81-92.

11. Stewart HJ, Horne GA, Bastow S and Chevassut TJ. BRD4 associates with p53 in DNMT3A-mutated leukemia cells and is implicated in apoptosis by the bromodomain inhibitor JQ1. Cancer medicine. 2013; 2:826-835.

12. Gallagher SJ, Mijatov B, Gunatilake D, Tiffen JC, Gowrishankar K, Jin L, Pupo GM, Cullinane C, Prinjha RK, Smithers N, McArthur GA, Rizos H and Hersey P. The epigenetic regulator I-BET151 induces BIM-dependent apoptosis and cell cycle arrest of human melanoma cells. J Invest Dermatol. 2014; 134:2795-2805.

13. Boi M, Gaudio E, Bonetti P, Kwee I, Bernasconi E, Tarantelli C, Rinaldi A, Testoni M, Cascione L, Ponzoni M, Mensah AA, Stathis A, Stussi G, Riveiro ME, Herait $\mathrm{P}$, Inghirami $\mathrm{G}$, et al. The BET Bromodomain Inhibitor OTX015 Affects Pathogenetic Pathways in Preclinical B-cell Tumor Models and Synergizes with Targeted Drugs. Clin Cancer Res. 2015; 21:1628-1638.

14. Shimamura T, Chen Z, Soucheray M, Carretero J, Kikuchi E, Tchaicha JH, Gao Y, Cheng KA, Cohoon TJ, Qi J, Akbay E, Kimmelman AC, Kung AL, Bradner JE and Wong KK. Efficacy of BET bromodomain inhibition in Kras-mutant non-small cell lung cancer. Clin Cancer Res. 2013; 19:6183-6192.

15. Bhadury J, Nilsson LM, Muralidharan SV, Green LC, Li Z, Gesner EM, Hansen HC, Keller UB, McLure KG and Nilsson JA. BET and HDAC inhibitors induce similar genes 
and biological effects and synergize to kill in Myc-induced murine lymphoma. Proc Natl Acad Sci U S A. 2014; 111:E2721-2730.

16. Fiskus W, Sharma S, Qi J, Valenta JA, Schaub LJ, Shah B, Peth K, Portier BP, Rodriguez M, Devaraj SG, Zhan M, Sheng J, Iyer SP, Bradner JE and Bhalla KN. Highly active combination of BRD4 antagonist and histone deacetylase inhibitor against human acute myelogenous leukemia cells. Mol Cancer Ther. 2014; 13:1142-1154.

17. Lee DH, Qi J, Bradner JE, Said JW, Doan NB, Forscher C, Yang H and Koeffler HP. Synergistic effect of JQ1 and rapamycin for treatment of human osteosarcoma. Int $\mathrm{J}$ Cancer. 2015; 136:2055-2064.

18. Wong C, Laddha SV, Tang L, Vosburgh E, Levine AJ, Normant E, Sandy P, Harris CR, Chan CS and Xu EY. The bromodomain and extra-terminal inhibitor CPI203 enhances the antiproliferative effects of rapamycin on human neuroendocrine tumors. Cell Death Dis. 2014; 5:e1450.

19. Tinsley S, Meja K, Shepherd C and Khwaja A. Synergistic induction of cell death in haematological malignancies by combined phosphoinositide-3-kinase and BET bromodomain inhibition. Br J Haematol. 2015; 170:275-8.

20. Abdulghani J and El-Deiry WS. TRAIL receptor signaling and therapeutics. Expert opinion on therapeutic targets. 2010; 14:1091-1108.

21. Bellail AC, Qi L, Mulligan P, Chhabra V and Hao C. TRAIL agonists on clinical trials for cancer therapy: the promises and the challenges. Rev Recent Clin Trials. 2009; 4:34-41.

22. Lim B, Allen JE, Prabhu VV, Talekar MK, Finnberg NK and El-Deiry WS. Targeting TRAIL in the treatment of cancer: new developments. Expert opinion on therapeutic targets. 2015:1-15.

23. Lemke J, von Karstedt S, Zinngrebe J and Walczak H. Getting TRAIL back on track for cancer therapy. Cell Death Differ. 2014; 21:1350-1364.

24. Wajant H. Targeting the FLICE Inhibitory Protein (FLIP) in cancer therapy. Mol Interv. 2003; 3:124-127.

25. Bagnoli M, Canevari S and Mezzanzanica D. Cellular FLICE-inhibitory protein (c-FLIP) signalling: a key regulator of receptor-mediated apoptosis in physiologic context and in cancer. Int J Biochem Cell Biol. 2010; 42:210-213.

26. Shirley $\mathrm{S}$ and Micheau O. Targeting c-FLIP in cancer. Cancer Lett. 2013; 332:141-50.

27. Kim Y, Suh N, Sporn M and Reed JC. An inducible pathway for degradation of FLIP protein sensitizes tumor cells to TRAIL-induced apoptosis. J Biol Chem. 2002; 277:22320-22329.

28. Poukkula M, Kaunisto A, Hietakangas V, Denessiouk K, Katajamaki T, Johnson MS, Sistonen L and Eriksson JE. Rapid turnover of c-FLIPshort is determined by its unique C-terminal tail. J Biol Chem. 2005; 280:27345-27355.

29. Ricci MS, Jin Z, Dews M, Yu D, Thomas-Tikhonenko A,
Dicker DT and El-Deiry WS. Direct repression of FLIP expression by c-myc is a major determinant of TRAIL sensitivity. Mol Cell Biol. 2004; 24:8541-8555.

30. Zhang L and Fang B. Mechanisms of resistance to TRAILinduced apoptosis in cancer. Cancer Gene Ther. 2005; 12:228-237.

31. Shirley S and Micheau O. Targeting c-FLIP in cancer. Cancer Lett. 2013; 332:141-150.

32. Zhang G, Plotnikov AN, Rusinova E, Shen T, Morohashi K, Joshua J, Zeng L, Mujtaba S, Ohlmeyer M and Zhou MM. Structure-guided design of potent diazobenzene inhibitors for the BET bromodomains. J Med Chem. 2013; 56:92519264.

33. Noel JK IK, Ooike S, Sugahara K, Nakamura H, Daibata M. Development of the BET bromodomain inhibitor OTX015. Mol Cancer Ther. 2014; 12:C244.

34. Ren H, Chen M, Yue P, Tao H, Owonikoko TK, Ramalingam SS, Khuri FR and Sun SY. The combination of RAD001 and NVP-BKM120 synergistically inhibits the growth of lung cancer in vitro and in vivo. Cancer Lett. 2012; 325:139-146.

35. Liu X, Yue P, Schonthal AH, Khuri FR and Sun SY. Cellular FLICE-inhibitory protein down-regulation contributes to celecoxib-induced apoptosis in human lung cancer cells. Cancer Res. 2006; 66:11115-11119.

36. Raja SM, Chen S, Yue P, Acker TM, Lefkove B, Arbiser JL, Khuri FR and Sun SY. The natural product honokiol preferentially inhibits cellular FLICE-inhibitory protein and augments death receptor-induced apoptosis. Mol Cancer Ther. 2008; 7:2212-2223.

37. Zou W, Chen S, Liu X, Yue P, Sporn MB, Khuri FR and Sun SY. c-FLIP downregulation contributes to apoptosis induction by the novel synthetic triterpenoid methyl-2cyano-3, 12-dioxooleana-1, 9-dien-28-oate (CDDO-Me) in human lung cancer cells. Cancer Biol Ther. 2007; 6:16141620 .

38. Zou W, Liu X, Yue P, Khuri FR and Sun SY. PPARgamma Ligands Enhance TRAIL-induced Apoptosis through DR5 Upregulation and c-FLIP Downregulation in Human Lung Cancer Cells. Cancer Biol Ther. 2007; 6:99-106.

39. Sun SY, Yue P, Dawson MI, Shroot B, Michel S, Lamph WW, Heyman RA, Teng M, Chandraratna RA, Shudo K, Hong WK and Lotan R. Differential effects of synthetic nuclear retinoid receptor-selective retinoids on the growth of human non-small cell lung carcinoma cells. Cancer Res. 1997; 57:4931-4939.

40. Liu X, Yue P, Zhou Z, Khuri FR and Sun SY. Death receptor regulation and celecoxib-induced apoptosis in human lung cancer cells. J Natl Cancer Inst. 2004; 96:17691780 . 